
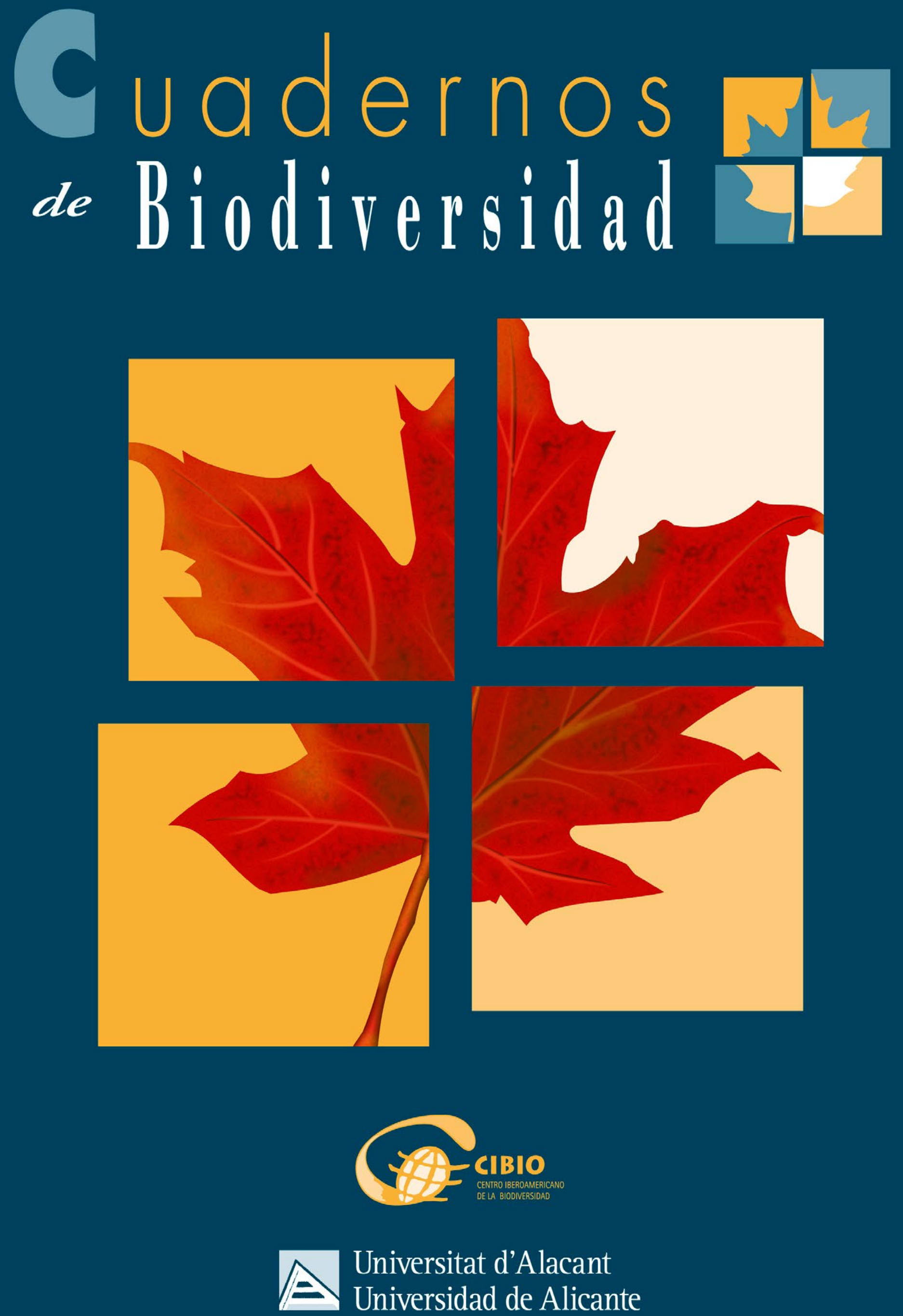


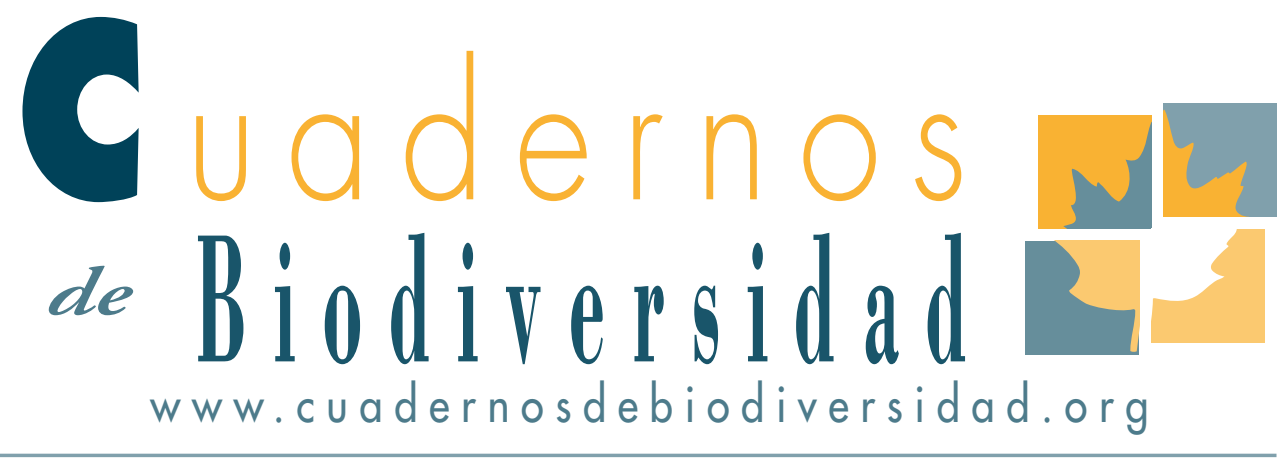

\section{Diversidad de sírfidos (Diptera: Syrphidae) de la Estación Biológica de Torretes (Alicante, España)}

The hoverfly diversity (Diptera: Syrphidae) of Torretes Biological Station (Alicante, Spain)

\section{E. M. Sánchez Heredia ${ }^{\mathrm{I}}$, N. Aniorte ${ }^{2}$, A. Ricarte ${ }^{3}$ y M. A. Marcos-García ${ }^{3}$}

i Calle Maestro Cabañes Ballester No 44 Onil (Alicante, España)

2 C/ Diagonal, Nº 12,3 B, Elche (Alicante, España)

3 Instituto de Investigación CiBiO, Universidad de Alicante. Crta. San Vicente del Raspeig S/N (Alicante, España)

ESTEBANI6_ONIL@HOTMAIL.COM,NURIA_AM92@HOTMAIL.COM, RICARTE24@GMAIL.COM, MARCOS@UA.ES

\section{RESUMEN}

Los sírfidos, o moscas de las flores, son una de las familias de dípteros que presenta una mayor diversidad biológica, por lo que su estudio nos aporta información muy valiosa para su aplicación en la conservación de los hábitats. Los adultos son asiduos visitantes florales, lo que les otorga un gran potencial como polinizadores, y la diversidad de regímenes tróficos de sus larvas hace que participen en diversos e importantes servicios ecosistémicos. En la base de cualquier estudio sobre ellos se encuentra el conocimiento de los géneros y especies que constituyen la comunidad de sírfidos de un ecosistema determinado, en nuestro caso, de los hábitats que conforman la Estación Biológica de Torretes, ubicada en el término municipal de Ibi (Alicante). Este conocimiento sentará las bases y facilitará la realización de posteriores estudios sobre interacciones biológicas que se establecen entre los sírfidos, sus 
plantas nutricias (polinización y herbivoría) y sus presas (control biológico).

\section{ABSTRACT}

Hoverflies, or flowerflies, are one of the most diverse Diptera families in biological terms, so their study provides a very valuable information to be applied for habitat conservation. Adults are frequent flower visitors, giving them great potential as pollinators, and the variation of trophic regimes in their larvae makes them to participate in important ecosystem services. On the bases of any hoverfly study is the knowledge of the genera and species that make up the hoverfly community of a given ecosystem, in our case, the habitats that make up the Biological Station Torretes, located in Ibi, Alicante province. This knowledge will establish the bases and facilitate further studies on biological interactions among hoverflies, their food plants (pollination and herbivory) and their preys (biological control).

Pallabras clave: sírfidos, área protegida, ecosistema mediterráneo.

Key words: hoverflies, protected area, Mediterranean habitats.

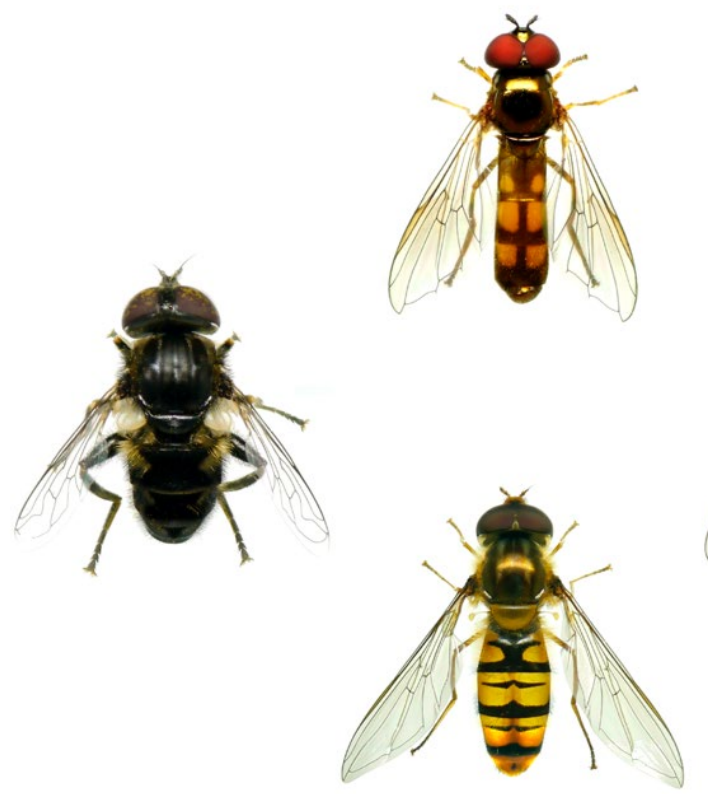

\section{INTRODUCCIÓN}

Los sírfidos (Diptera: Syrphidae) son una familia de dípteros ampliamente distribuida por todas las regiones geográficas, excepto en la Antártida, y representada por unas 6.000 especies a nivel mundial. En España se conocen 417 especies (Ricarte \& Marcos-García, 2017), aunque aún quedan muchas áreas por explorar, tanto desde el punto de vista faunístico, como de la biología de sus especies. La obra de Rotheray \& Gilbert (2011) ha facilitado enormemente la difusión del conocimiento a cerca de estos dípteros. En su fase adulta, poseen una gran variabilidad morfológica, cromática y de tamaño, mimetizando con cierta frecuencia a especies de Himenópteros y de otras familias de Dípteros (Fig. 1). Los adultos se caracterizan por su vuelo rápido y cernido (Rotheray \& Gilbert, 2011) y son frecuentes visitadores de flores, lo que en ocasiones les convierte en buenos polinizadores. En su fase larvaria presentan una gran diversidad de regímenes tróficos y hábitos de vida, siendo principalmente saprófagos, depredadores y fitófagos (Brauns, 1953), lo que nos muestra con claridad su activa participación en diversos procesos biológicos de los ecosistemas, por lo que son buenos indicadores de su estado de conservación (Marcos-García \& Ricarte, 2009; Ricarte et al., 2011). Debido a esto, cualquier cambio o alteración en el entorno, puede afectar a la composición de las comunidades de sírfidos que en él se encuentren (Sommaggio, 1999).

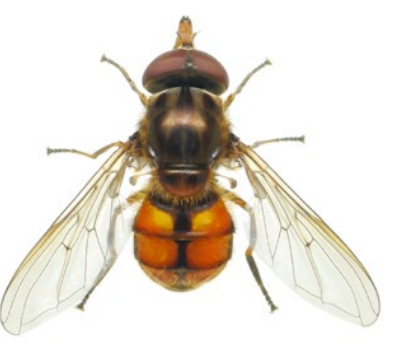

Figura 1. Ilustración de distintos géneros de sírfidos, mostrando su variedad morfológica (Speight $\&$ de Courcy Williams, 2016). 
Las especies con larvas depredadoras desempeñan una función muy importante en el ámbito agrícola, ya que actúan como agentes reguladores naturales de insectos que ocasionan plagas. Estas larvas se alimentan principalmente de homópteros de cuerpo blando, pero también de otros insectos como larvas de Lepidópteros y Coleópteros (Rojo et al., 2003). Las larvas de unas pocas especies fitófagas se alimentan de bulbos de Liliáceas y Amarillidáceas (Ricarte et al., 2008), pudiendo ocasionar pérdidas económicas en cultivos comerciales de especies ornamentales como los narcisos. Podemos observar a los adultos con cierta frecuencia volando en jardines urbanos, entornos rurales (Sommaggio, 1999), ecosistemas agrícolas (Pineda \& Marcos-García, 2008) y principalmente, en medios forestales (Ricarte, 2008).

Por las razones expuestas, el conocimiento de las especies de sírfidos que hay en cualquier ecosistema, puede indicarnos en cierto modo, su estado de conservación. Trabajos como Pollard (1971) y Speight y Castella (2001), en los que se proponen estrategias y medidas de conservación en los ecosistemas, ejemplifican cómo el estudio de la biodiversidad de sírfidos es de gran importancia a estos efectos.

Este trabajo se ha realizado en La Estación Biológica/Jardín Botánico de Torretes, un ecosistema mediterráneo en mosaico de la provincia de Alicante (España), con una elevada diversidad de flora y fauna que la han hecho merecedora de varias figuras de protección.

No se ha llevado a cabo hasta el momento ningún estudio sobre la entomofauna de este área natural, siendo nuestro principal objetivo conocer las especies de sírfidos que viven y se desarrollan en la Estación Biológica de Torretes y su distribución a lo largo del año.

\section{MATERIAL Y MÉTODOS}

El trabajo de campo ha sido realizado en la Estación Biológica de Torretes, localizada en el término municipal de Ibi (Alicante). La Estación tiene una superficie de $53 \mathrm{Ha}$, de las cuales $44 \mathrm{Ha}$ corresponden a superficie forestal y $9 \mathrm{Ha}$ a superficie agraria. Abarca de los 900 a los 1100 metros de altitud, y se sitúa en la cara Sur del Parque Natural del Carrascal de la Font Roja, entre los $30^{\circ}$ y los $40^{\circ}$ de latitud. Se suelen dar tres meses de sequía estival y heladas ocasionales durante el invierno (Ríos \& MartínezFrancés, 2007). Sus precipitaciones se encuentran entre los 250 y los $1500 \mathrm{~mm}$, son estacionalmente irregulares y con frecuenciase producen fenómenos de torrencialidad (Alcaraz, 1999).

La Estación funciona como centro de investigación y divulgación científica adscrito al Instituto Universitario de Investigación (CIBIO) de la Universidad de Alicante, que tiene, como uno de sus objetivos principales, difundir conocimiento a favor de la Conservación de la Naturaleza.

El material estudiado se ha recolectado en el periodo de tiempo comprendido entre noviembre de 2015 y abril de 2016. Además, se ha incluido también material recolectado por los autores durante los ańos 2014 y 2015.

El método de muestreo empleado consistió principalmente en trampas Malaise (Townes, 1962 modelo modificado) (Fig.2), situadas en tres puntos estratégicos de la Estación Biológica de Torretes. Para la elección de estos puntos se seleccionaron hábitats lo suficientemente diferenciados con el fin de facilitar, en la medida de lo posible, la captura del mayor número de especies que constituyen la comunidad de sírfidos de la Estación.

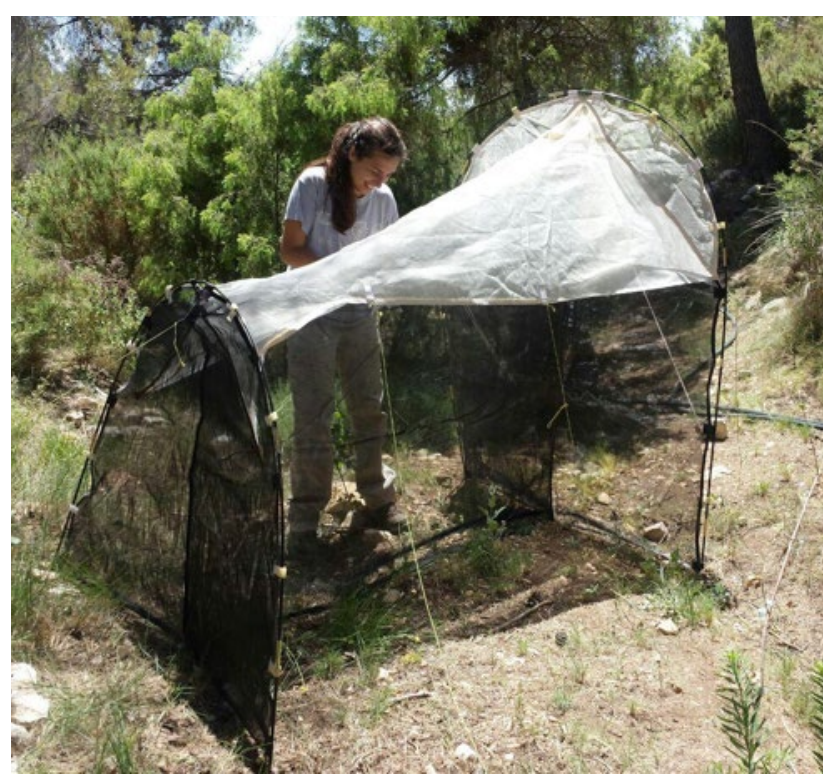

Figura 2. Trampa Malaise tipo Townes modificada. 


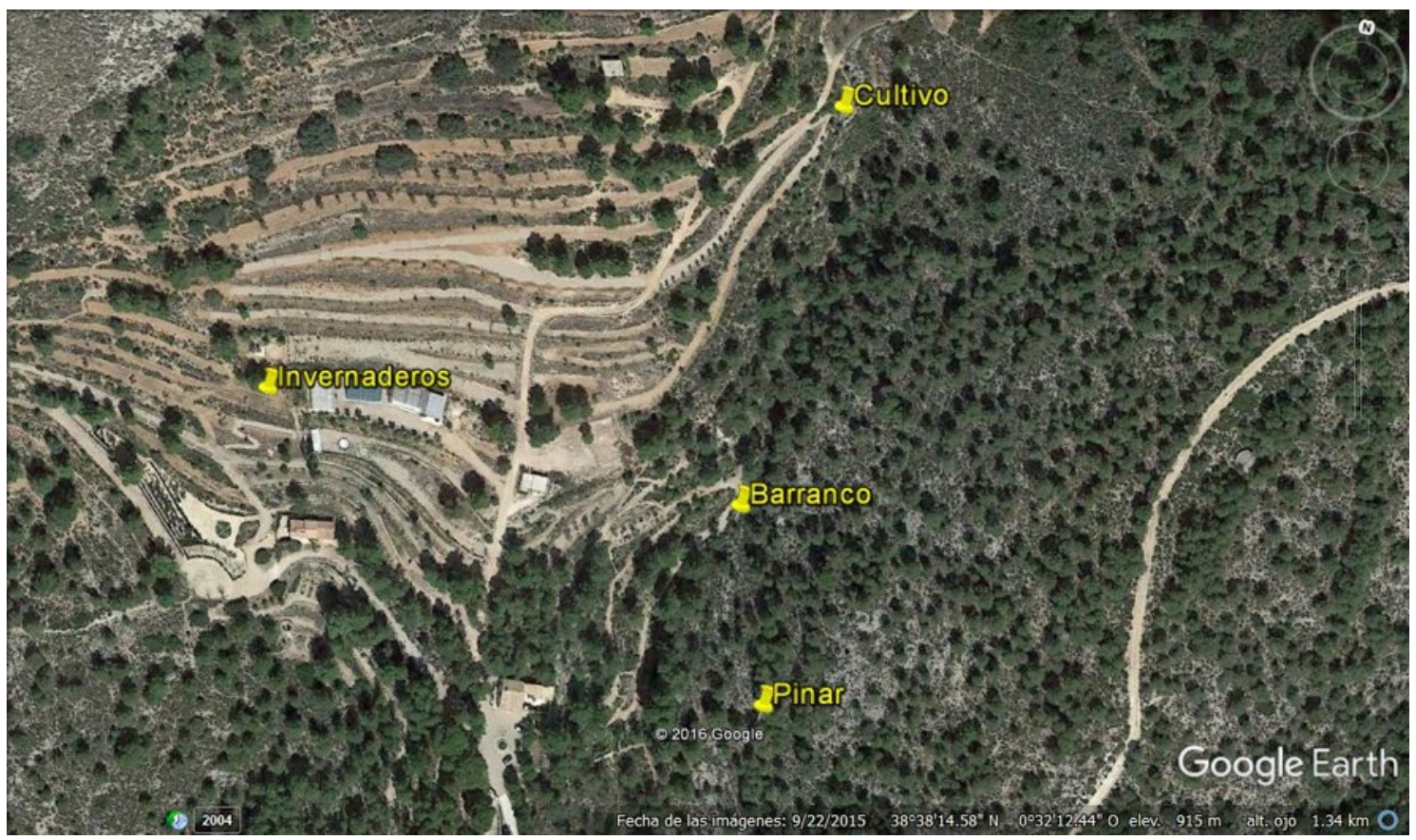

Figura 3. Situación de las trampas Malaise utilizadas.

Las trampas Malaise recolectan por intercepción insectos voladores que, una vez atrapados, se dirigen a un recipiente recolector donde permanecen conservados hasta su cambio y recogida quincenal. Suelen colocarse en áreas de paso que actúan como corredores de vuelo de los insectos, de modo que estos acaben accediendo por los laterales de la trampa hasta entrar en el recipiente con el líquido conservante, que es alcohol de $70^{\circ}$ más glicerol, con el fin de evitar la evaporación y la congelación.

También se realizaron muestreos complementarios con manga entomológica, ya que no todas las especies son susceptibles de ser capturadas con la misma probabilidad mediante las trampas Malaise (Marcos-García et al., 2012). Se seleccionaron lugares específicos para el mangueo, con características favorables para la presencia de sírfidos, como la cercanía a puntos de agua o la abundancia de flores blancas y amarillas, colores a los que los sírfidos adultos acuden con asiduidad.

Para la ubicación de las trampas, se consideraron tres ambientes distintos caracterizados por la vegetación circundante. De este modo, distinguimos tres zonas principales, más una cuarta adicional (Fig. 3):
Zona de Invernaderos: Denominada así debido a su proximidad a los invernaderos de la Estación Biológica. Es un área de antiguos bancales situada en la solana, con predominio de gramíneas y escasa cobertura vegetal. Se localiza a 913 m.s.n.m. y situada al Oeste de la Estación.

Zona de Cultivos: Se trata de una zona circundada por bancales de almendros y otros árboles frutales con predominio de vegetación herbácea ruderal y ausencia de matorral. Se encuentra a 936 m.s.n.m. y situada al Norte de la Estación.

Zona de Barranco: Es una zona situada en el fondo de un pequeño barranco, una de cuyas laderas conduce a un bosquete de Pinus halepensis. Es una zona con predominio de vegetación herbácea de ribera, protegida del viento y cercana a puntos de acúmulo de agua, lo que le convierte en ruta de paso y punto de atracción para muchos insectos. Se encuentra a una altura de 918 metros.

Zona de pinar: Además se contó con datos de ejemplares de sírfidos muestreados de mayo a diciembre de 2014 en una zona de Pinus halepensis localizada a 906 m.s.n.m. y orientada al Este de la Estación. 


\section{IDENTIFICACIÓN DEL MATERIAL}

La identificación se llevó a cabo a nivel de especie, requiriéndose en algunos casos el examen de la estructura genital masculina. Para su identificación a nivel de género se empleó la obra de Van Veen (2004) y para la identificación a nivel de especie, se requirió bibliografía específica de cada género. La información sobre distribución y biología larvaria de las especies procede de Speight (2015).

Todo el material recolectado forma parte de la colección entomológica de la Universidad de Alicante (CEUA), depositada en el CIBIO.

\section{RESULTADOS}

En la Tabla 1 se pueden observar las especies estudiadas, su fenología y el número de machos y hembras obtenido.

Tabla 1. Listado de especies de sírfidos recolectadas y su fenología. Cuando una especie solo se ha registrado en una quincena del mes, se colorea, tan solo, la mitad correspondiente de la celda. Leyenda: $\mathrm{B}=$ Barranco (incluye la zona de la charca en torno a la cual se capturaron numerosos sírfidos con manga entomológica); $\mathrm{C}=$ Cultivo; $\mathrm{I}=$ Invernaderos; $\mathrm{P}=$ Pinar $; \mathrm{M}=$ Trampa Malaise; $\mathrm{m}=$ Manga entomológica.

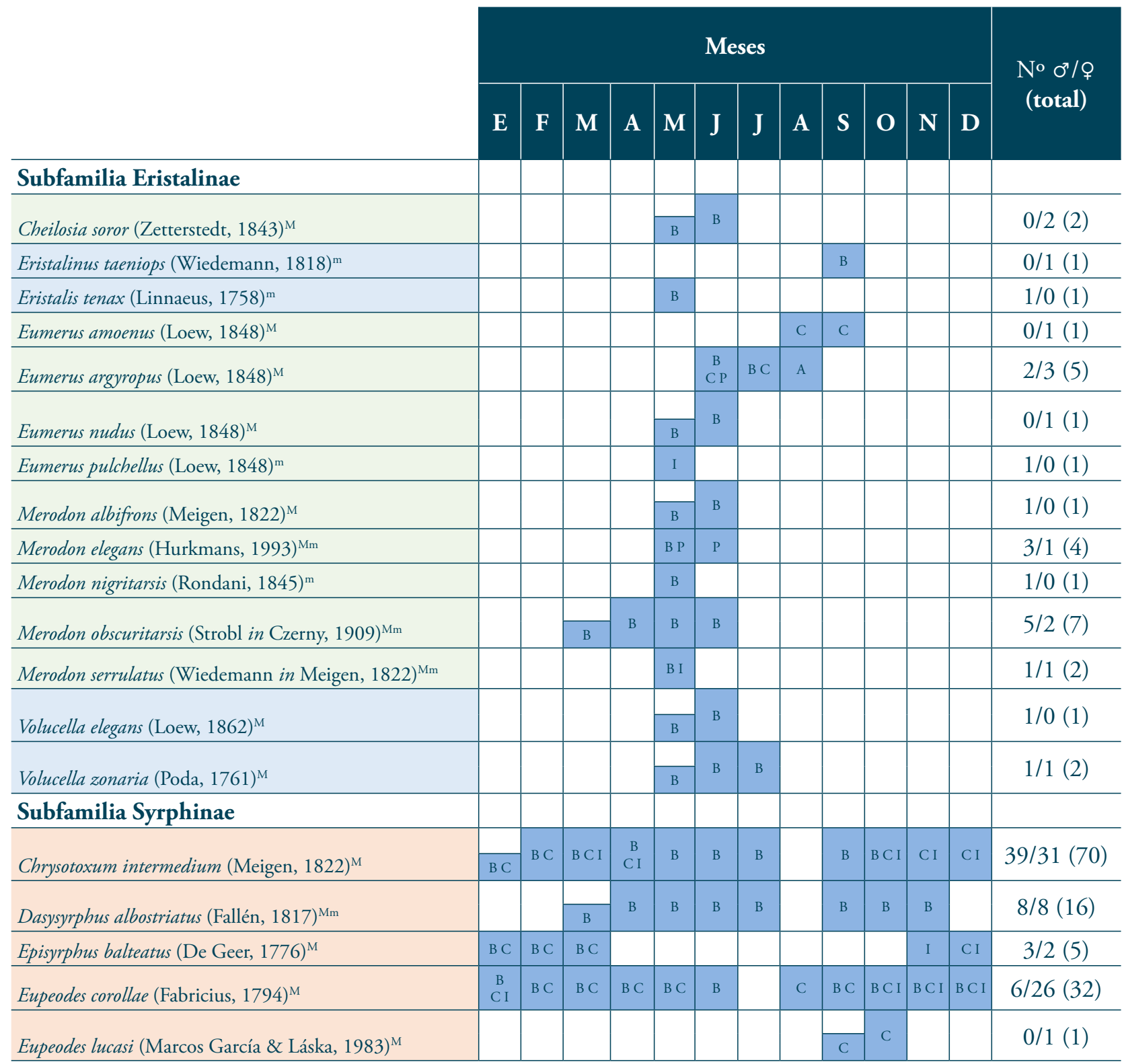




\begin{tabular}{|c|c|c|c|c|c|c|c|c|c|c|c|c|c|}
\hline Eupeodes luniger (Meigen, 1822) ${ }^{\mathrm{M}}$ & & & & & & B & B & & & & & & $0 / 1(1)$ \\
\hline Eupeodes nielseni (Dušek \& Láska, 1976) ${ }^{\mathrm{M}}$ & & & $\mathrm{C}$ & C & & & & & & & & & $0 / 1(1)$ \\
\hline Melanostoma mellinum (Linnaeus, 1758$)^{\mathrm{M}}$ & & B & $\mathrm{BC}$ & C & B & B & & & & & & & $1 / 3(4)$ \\
\hline Meliscaeva auricollis (Meigen, 1822) ${ }^{\mathrm{M}}$ & в С & $\mathrm{BC}$ & В C & B C & в & в & B & & & & I & B I & $15 / 3(18)$ \\
\hline Paragus bicolor (Fabricius, 1794) ${ }^{\mathrm{Mm}}$ & & & & & BI & $\mathrm{BCI}$ & В I & в І & 1 & I & & & $6 / 10(16)$ \\
\hline Paragus flammeus (Goeldlin, 1971) ${ }^{\mathrm{M}}$ & & & & & B & B & & & & & & & $0 / 2(2)$ \\
\hline Paragus quadrifasciatus (Meigen, 1822) ${ }^{\mathrm{M}}$ & & & & & BI & BC & BC & & & & & & $1 / 8(9)$ \\
\hline Paragus strigatus (Meigen, 1822) ${ }^{\mathrm{M}}$ & & & & & B & в & & & & & & & $0 / 1(1)$ \\
\hline Paragus tibialis (Fallén, 1817) ${ }^{\mathrm{Mm}}$ & & & & & I & в & в & & & & & & $2 / 5(7)$ \\
\hline Pelecocera pruinosomaculata $(\text { Strobl, } 1906)^{\mathrm{M}}$ & & & В & B & & $\mathrm{P}$ & & & $\begin{array}{l}\mathrm{BC} \\
\mathrm{PI} \\
\end{array}$ & $\begin{array}{c}\text { B C } \\
\text { P I } \\
\end{array}$ & $\begin{array}{c}\mathrm{B} \\
\mathrm{CP}\end{array}$ & в С & $\begin{array}{c}73 / 120 \\
(193) \\
\end{array}$ \\
\hline Platycheirus splendidus (Rotheray, 1998) ${ }^{\mathrm{M}}$ & & & $\mathrm{B}$ & в & в & в & & & & & & & $2 / 0(2)$ \\
\hline Scaeva mecogramma $(\text { Bigot, } 1860)^{\mathrm{M}}$ & & & & & & в & в & & & & & & $1 / 1(2)$ \\
\hline Scaeva pyrastri $(\text { Linnaeus, } 1758)^{\mathrm{m}}$ & & & & & & в & & & & & & & $1 / 0(1)$ \\
\hline Scaeva selenitica (Meigen, $1822 \mathrm{M}$ & & & & & & C & $\mathrm{C}$ & & & & & & $0 / 1(1)$ \\
\hline Sphaerophoria scripta (Linnaeus, 1758) ${ }^{\mathrm{M}}$ & & $\mathrm{BC}$ & В C & C & B I & в & в & & & & & & $6 / 8(14)$ \\
\hline Syrphus vitripennis (Meigen, 1822) ${ }^{\mathrm{m}}$ & & & & & I & & & & & & & & $1 / 0(1)$ \\
\hline Xanthogramma dives (Rondani, 1857) ${ }^{\mathrm{M}}$ & & & & & & в & в & & & & & & $1 / 0(1)$ \\
\hline Xanthogramma marginale (Loew, 1854) ${ }^{\mathrm{M}}$ & & & & & $\mathrm{I}$ & & & & c & c & & & $0 / 2(2)$ \\
\hline
\end{tabular}

La siguiente leyenda indica la correspondencia entre el color asignado en la tabla y la biología larvaria de la especie en cuestión:

Biología larvaria saprófaga

Biología larvaria fitófaga

Biología larvaria depredadora

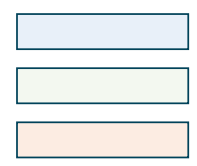

En la Tabla 2 se representa el número de especies y de ejemplares de sírfidos por hábitat muestreado.

\begin{tabular}{l|c|c}
\hline Zona de muestreo & Número de especies & Número de individuos \\
\hline Barranco & 30 & 276 \\
\hline Cultivo & 14 & 75 \\
\hline Pinar & 3 & 48 \\
\hline Invernadero & 11 & 37
\end{tabular}

Tabla 2. Número recolectado de especies e individuos totales de sírfidos para cada una de las cuatro zonas de muestreo estudiadas. 


\section{COMENTARIOS FINALES}

En total, se han estudiado 37 especies de sírfidos (62.16\% depredadoras, $27.02 \%$ fitófagas y $10.81 \%$ saprófagas).

Se cita, por primera vez en la Península Ibérica, la especie Platycheirus splendidus. Esta especie ha sido confundida con especies afines, por lo que el conocimiento sobre su distribución geográfica es impreciso. Hasta el momento esta especie, también presente en la región Neártica, se conoce de países europeos como Irlanda, Gran Bretańa, Escandinavia, Bélgica y Alemania, hasta los Alpes (Speight, 2015). Se citan también, por vez primera para la provincia de Alicante, las especies: Eupeodes nielseni, Paragus flammeus, Pelecocera pruinosomaculata y Xanthogramma dives (Ricarte \& Marcos-García (2017) y se confirma la presencia en la zona de Merodon serrulatus, especie endémica de la Península Ibérica (Marcos García et al., 2007).

Se destaca el alto número de ejemplares (193 individuos) recolectados de Pelecocera pruinosomaculata a lo largo de todo el año excepto en los meses más cálidos y más fríos y de Chrysotoxum intermedium (70 individuos), que vuela a lo largo de todo el año, excepto en agosto.

Se ha estudiado un mayor número de especies pertenecientes a los géneros Paragus, Eumeurus y Merodon, resultados lógicos dado que estos tres géneros presentan una distribución mediterránea. Por otro lado, se destaca también la presencia de una única especie del género Cheilosia, mejor representado en la región eurosiberiana ibérica (Ricarte $\&$ Marcos-García, 2017).

En cuanto a la abundancia de ejemplares, es durante la primavera cuando se detecta el mayor número de especies y de ejemplares de sírfidos (Tabla 1).

Se constata una mayor presencia de sírfidos en la zona del barranco (62\%, 276 individuos), seguida de la zona de cultivo (18\%, 75 individuos), pinar (12\%, 48 individuos) e invernaderos (8\%, 37 individuos). La zona del pinar es la de menor riqueza, habiéndose recolectado sólo 3 especies.
La trampa Malaise situada en la zona del barranco se encontraba próxima a una fuente de agua, donde también se recolectó una gran cantidad de sírfidos con manga entomológica. Esto se debe a que el barranco actúa de corredor de vuelo y, en este punto de agua, los adultos se detienen a hidratarse y alimentarse del polen y néctar de las flores que crecen en la periferia de la fuente, convirtiéndose este lugar en un importante punto de encuentro para los sírfidos adultos.

Las especies de sírfidos que presentan una biología larvaria depredadora juegan un papel importante en el control natural de plagas agrícolas, desarrollándose en esta área natural 23 especies de 13 géneros que, sin duda, están ejerciendo un control natural en las poblaciones de homópteros plaga de los cultivos cercanos.

La destacada abundancia de Pelecocera pruinosomaculata en la Estación Biológica de Torretes, parece indicar que se dan las adecuadas condiciones para el desarrollo de su ciclo biológico, que es aún desconocido. De las especies estudiadas también se desconoce el ciclo biológico de Cheilosia soror, Eumerus argyropus, Eupeodes lucasi, Merodon albifrons, Merodon elegans, Merodon nigritarsis, Merodon obscuritarsis, Merodon serrulatus, Paragus bicolor, Paragus flammeus, Paragus strigatus, Paragus tibialis, Volucella elegans, Xanthogramma dives y Xanthogramma marginale (Speight, 2015).

Estos resultados animan a realizar nuevos estudios puntuales en la zona dirigidos al descubrimiento del microhábitat donde se desarrollan las fases larvarias de estas especies y las condiciones de sus ciclos de desarrollo, con el fin de poder valorar el estado de conservación de cada hábitat dentro de la Estación Biológica de Torretes. A partir de esta información, podremos definir las estrategias adecuadas para el mantenimiento de la diversidad de estas especies bioindicadoras responsables de diferentes funciones ecosistémicas.

\section{AGRADECIMIEMTOS}

Agradecemos a Anita Nencioni, Cinta Quirce y Roberto Poyatos por su ayuda con el mantenimiento de las trampas Malaise. 


\section{REFERENCIAS}

Abelló, P., Barrientos, J.A. (2004). Curso Práctico de Entomología. Asociación Española de Entomología, CIBIO: Centro Iberoamericano de la Biodiversidad (Alicante), Universitat Autónoma de Barcelona. Bellaterra, Alicante.

Alcaraz, F.J. (1999). Manual de teoría y práctica de Geobotánica. Eds. DM, ICE, Universidad de Murcia. 401 pp.

Brauns, A. (1953). Studies on the ecology and economic importance of aphidophagous species of Syrphidae. Beitr. Ent., 3: 278-303.

Marcos-García, M.A., Vujić, A. \& Mengual, X. (2007). Revision of Iberian species of the genus Merodon Meigen, 1803 (Diptera: Syrphidae). European Journal of Entomology 104 (3): 531-572.

Marcos-García, M.A., García-López, A., Zumbado, M.A. \& Rotheray, G. E. (2012). Sampling Methods for Assessing Syrphid Biodiversity (Diptera: Syrphidae) in Tropical Forests. Environmental Entomology 41(6): 1544«1552 (2012); DOI: http://dx.doi.org/10.1603/EN12215.

Marcos-García, M.A. \& Ricarte, A. (2009). Los sírfidos (Diptera: Syrphidae) saproxílicos como indicadores del estado de conservación del Parque Nacional de Cabañeros: 201-21. Proyectos de Investigación en Parques Nacionales: 2005-2008., Ramírez L. y Asencio B., ed. Organismo Autónomo Parques Nacionales. Ministerio del Medio Ambiente.

Pineda, A., Marcos-García, M.A. (2008). Seasonal abundance of aphidophagous hoverflies (Diptera: Syrphidae) and their populational levels in- and outside of Mediterranean sweet-pepper greenhouses. Annals of the Entomological Society of America, 101 (2):384-391.

Pollard, E. (1971). Hedges VI. Habitat diversity and crop pests: a study of Brevicoryne brassicae and its syrphid predators. J. Appl. Ecol., 8: 751-780.

Ricarte, A. (2008). Biodiversidad de sírfidos (Diptera: Syrphidae) y conservación de los hábitats en el Parque Nacional de Cabañeros, España. Universidad de Alicante. 289 pp.

Ricarte, A., Marcos-García, M.A. \& Rotheray G.E. (2008). The early stages and life histories of three Eumerus and two Merodon species (Diptera: Syrphidae) from the Mediterranean region. Entomologica Fennica 19: 129-141.
Ricarte, A., Moreno, C. \& Marcos-García, M.A. (2011). Assessing the effects of vegetation type on hoverfly (Diptera: Syrphidae) diversity in a Mediterranean landscape: implications for conservation. Journal of Insect Conservation, 15: 865-877.DOI 10.1007/s10841-011-9384-9.

Ricarte, A., Quinto, J., Speight, M.C.D. \& Marcos-García, M.-A. (2013). A contribution to knowledge of the biodiversity of Syrphidae (Diptera) in Spain. Arch. Biol. Sci., Belgrade, 65: 1533-1537.

Ricarte, A., Marcos-García, M. A. (2017). A checklist of the Syrphidae (Diptera) of Spain, Andorra and Gibraltar. Zootaxa, 4216 (5): 401-440.

Ríos, S. \& Martínez-Francés, V. (2007). El Jardín Etnobotánico de la Estación Biológica de Torretes-Font Roja, CIBIO-Universidad de Alicante. Cuadernos de biodiversidad. No 23 (jun. 2007), p. 21.

Rojo, S., Gilbert, F., Marcos-García, M.A., Nieto, J.M. \& Mier, M.P. (2003). A World Review of Predatory Hoverflies (Diptera, Syrphidae: Syrphinae) an their Prey. CIBIO Ediciones, Alicante, 319 pp.

Rotheray, G.E. \& Gilbert, F.S. (2011). The Natural History of Hoverflies. Forrest Text, Cardigan. 333 pp.

Sommaggio D. (1999). Syrphidae: can they be used as environmental bioindicators? Agriculture Ecosystems \& Environment 74:343-356.

Speight, M.C.D. (2015). Syrph the net: the database of biological information about European Syrphidae (Diptera) and its use in relation to the conservation of biodiversity. En: B. S. Rushton (Eds.). Biodiversity: the Irish dimension. Royal Irish Academy, Dublin.

Speight, M.C.D. \& Castella, E. (2001). An approach to interpretation of lists of insects using digitised biological information about the species. Journal of Insect Conservation 5:131-139.

Speight, M.C.D. \& de Courcy Williams, M. (2016) European Syrphid Genera: Portraits of representative species/ Portraits d'espèces représentatives de genres de Syrphidae Européens. Syrph the Net, the database of European Syrphidae (Diptera), Vol. 89, 72 pp, Syrph the Net publications, Dublin.

Townes, H. (1962). Design for a Malaise trap. Proceeding of the Entomological Society Washington. 64(4), 253-262.

Van Veen, M.P. (2004). Hoverflies of northwest Europe. Identification keys to the Syrphidae. KNNV Uitgeverij, Utrecht. 